\title{
Synthetic lethality in malignant pleural mesothelioma with PARP1 inhibition
}

\author{
Gayathri Srinivasan $^{1}$ - Gurjit Singh Sidhu ${ }^{1}$ Elizabeth A. Williamson ${ }^{1}$ • \\ Aruna S. Jaiswal ${ }^{1} \cdot$ Nasreen Najmunnisa $^{1} \cdot$ Keith Wilcoxen $^{2} \cdot$ Dennie Jones $^{1}$ • \\ Thomas J. George $\mathbf{J r}^{1} \cdot$ Robert Hromas $^{1}$ (i)
}

Received: 13 March 2017 / Accepted: 21 July 2017 / Published online: 29 July 2017

(c) The Author(s) 2017. This article is an open access publication

\begin{abstract}
Malignant pleural mesotheliomas (MPM) are most often surgically unresectable, and they respond poorly to current chemotherapy and radiation therapy. Between 23 and $64 \%$ of malignant pleural mesothelioma have somatic inactivating mutations in the BAP1 gene. BAP1 is a homologous recombination (HR) DNA repair component found in the BRCA1/BARD1 complex. Similar to BRCA1/2 deficient cancers, mutation in the BAP1 gene leads to a deficient HR pathway and increases the reliance on other DNA repair pathways. We hypothesized that BAP1-mutant MPM would require PARP1 for survival, similar to the BRCA1/2 mutant breast and ovarian cancers. Therefore, we used the clinical PARP1 inhibitors niraparib and olaparib to assess whether they could induce synthetic lethality in MPM. Surprisingly, we found that all MPM cell lines examined, regardless of BAP1 status, were addicted to PARP1-mediated DNA repair for survival. We found that niraparib and olaparib exposure markedly decreased clonal survival in multiple MPM cell lines, with and without BAP1 mutations. This clonal cell death may be due to the extensive replication fork collapse and genomic instability that PARP1 inhibition induces in MPM cells. The requirement of MPM cells for PARP1
\end{abstract}

Electronic supplementary material The online version of this article (doi:10.1007/s00280-017-3401-y) contains supplementary material, which is available to authorized users.

Thomas J. George Jr.

thom.george@medicine.ufl.edu

Robert Hromas

rhromas@ufl.edu

1 Department of Medicine and the Cancer Center, University of Florida Health, 1600 SW Archer Rd, Gainesville, FL 32610, USA

2 Tesaro, Waltham, MA 02451, USA suggests that they may generally arise from defects in HR DNA repair. More importantly, these data demonstrate that the PARP1 inhibitors could be effective in the treatment of MPM, for which little effective therapy exists.

Keywords Mesothelioma $\cdot$ BAP1 PARP1 $\cdot$ Homologous recombination $\cdot$ Niraparib $\cdot$ Olaparib

\section{Introduction}

Malignant pleural mesothelioma (MPM) is a rare and aggressive tumor primarily caused by inhalation of asbestos. Treatment for MPM is most commonly surgery, with chemotherapy/radiotherapy for residual disease $[1,2]$. The prognosis for this disease is dismal, given that complete resection of the tumor is difficult, and the lack of sensitivity to chemotherapy or radiation [3]. In addition, response to newer agents, such as the anti-angiogenic biological therapies and the kinase targeted small molecules, has been disappointing [1,2].

Interestingly, between 23 and 64\% of MPMs have somatic inactivating mutations in the BAP1 (BRCA1-associated protein 1) gene [4-7]. BAP1 is also mutated in a fraction of uveal melanomas, renal cell carcinomas, and cholangiocarcinoma [8]. BAP1 is a member of the BRCA1/BARD1 complex and plays a role in homologous recombination (HR) DNA double strand break (DSB) repair $[9,10]$. BAP1 interacts with the BRCA1 RING finger domain, which mediates BRCA1's E3 ubiquitin ligase activity [11]. BAP1 functions as a deubiquitinase and BRCA1 likely recruits it to sites of DSBs to restore histone ubiquitination to native levels after repair is completed [11].

We hypothesized that BAP1-mutant mesothelioma might be addicted to PARP1-mediated DNA repair pathways, 
similar to the BRCA1/2-mutant breast and ovarian cancers [12-14]. BAP1-mutant MPM would have defects in the HR DNA repair, and thus may also require PARP1 for proliferation. PARP1 inhibition in BRCA1-deficient cancers results in collapsed replication forks and genomic instability leading to mitotic catastrophe and ultimately cell death [12, 13]. When a cancer arises from defects in one DNA repair pathway, such as HR in the BRCA1/2-mutant malignancies, they become addicted to other DNA repair pathways for survival during replication [12-14]. A well-known example of this is the inhibition of the DNA repair component PARP1 in the BRCA1-deficient cancers result in replication fork collapse and mitotic catastrophe [12-14]. In this situation, PARP1 inhibition stalls replication forks, which cannot be repaired because of the underlying defect in HR in these cancers [12]. Sensitivity to PARP1 inhibition is one biomarker of HR deficiency $[12,14]$.

Significantly, we discovered that the clinical PARP1 inhibitors, niraparib and olaparib, markedly decreased clonal survival in all MPM cell lines tested, regardless of BAP1 status. This implies that defects in homologous recombination DNA repair are at the origin of most MPM. More importantly, these data suggest a new modality for targeted therapy to be studied in a cancer that desperately needs effective treatment.

\section{Materials and methods}

\section{Cell culture, drug treatment and survival assay}

The BAP1-mutant MPM cell lines, H-Meso01, H2452, H2461, H2731, and the wild type BAP1 MPM cell lines CRL-2081 and H290 were cultured in RPMI 1640 medium supplemented with $10 \%$ fetal bovine serum, and $1 \%$ penicillin and streptomycin. Niraparib and olaparib were purchased from MedChem Express (Monmouth Junction, NJ). Clonal survival assays were performed as described [14]. All experiments were repeated at least three times in triplicate, and no data points were excluded from statistical analysis.

\section{Nuclear structure assays and DNA damage foci}

Nuclear structural abnormalities (micronuclei and bridging) arising from aberrant chromosomal segregation after replication fork fusion were assessed as we described [14]. Briefly, DMSO control and olaparib- or niraparib-treated cells were grown on coverslips, and the cells were fixed in $100 \%$ methanol at $-20{ }^{\circ} \mathrm{C}$ for $20 \mathrm{~min}$ for $72 \mathrm{~h}$ after niraparib treatment at $3 \mathrm{uM}$. The fixed cells were mounted using DAPI-Fluoromount $\mathrm{G}$ clear mounting media from SouthernBiotech and analyzed within $24 \mathrm{~h}$ with laser confocal scanning microscope (TCS-SP5, Leica Microsystems, Exton,
PA) as described [14]. At least six distinct determinations (100-150 nuclei per determination) were performed for each group.

Confocal Immunofluorescence foci assays were performed for $\gamma$-H2Ax DNA damage foci analysis as we described [14]. The $\gamma$-H2AX (S139) primary antibody was from Millipore (Billerica, MA), and the secondary antibody conjugated with Alexa Fluor dye was from Invitrogen (Waltham, MA). After staining, coverslips were mounted with DAPI-Fluoromount G clear mounting media from SouthernBiotech (Birmingham, AL) and analyzed within $24 \mathrm{~h}$ with a laser confocal scanning microscope (TCS-SP5). At least six distinct determinations (100-150 nuclei per determination) were performed for each condition for statistical analysis. Cells with more than five foci were counted as positive. Photomicrographs of each distinct cell population were taken at equal magnifications and equal fluorescence intensities.

\section{Replication fork restart analysis}

Replication fork restart after treatment with niraparib was measured using immunofluorescent detection of BrdU foci after DNA denaturation as described [14]. Briefly, after treatment in $3 \mu \mathrm{M}$ niraparib for $48 \mathrm{~h}$, cells were released into fresh media containing $10 \mathrm{uM}$ BrdU (BD Biosciences, Franklin Lakes, NJ, USA) for $30 \mathrm{~min}$. After washing, cells on coverslip were fixed and the DNA was denatured using hydrochloric acid for BrdU immunostaining. The coverslips were processed for immunostaining using BrdU-specific antibody (Cell Signaling, Danvers, MA, USA) and then incubated with a secondary antibody conjugated with Alexa Fluor dye from Invitrogen for $1 \mathrm{~h}$. Cells were analyzed as above using the laser scanning confocal microscope at the same magnification and the same fluorescence. At least 500 cells were counted for each condition from at least six distinct slides per condition for statistical analysis.

\section{Cytogenetic and sister chromatid exchange analysis}

Structural aberrations in metaphase chromosomes were scored after solid Giemsa staining as we described [14, 15]. Sister chromatid exchanges were analyzed using modifications from previously described protocols [16, 17]. The detailed modifications are as follows: Cells were treated with either $0.06 \%$ DMSO or $3 \mu \mathrm{M}$ of niraparib for $48 \mathrm{~h}$ along with BrdU at a final concentration of $5 \mu \mathrm{g} / \mathrm{mL}$. Forty-eight hours after the treatment, the cells were washed and replaced with fresh media without the drug or BrdU. To this, $10 \mu \mathrm{g} /$ mL of KaryoMAX colcemid stock (ThermoFisher Scientific, Waltham, MA, USA) was added to give a final concentration of $0.1 \mu \mathrm{g} / \mathrm{mL}$ and incubated for $1 \mathrm{~h}$ in the cell culture incubator. The cells were then washed with fresh media and 
$2 \mathrm{~mL}$ of pre-warmed $75 \mathrm{mM} \mathrm{KCl}$ was added to the cells and incubated at $37{ }^{\circ} \mathrm{C}$ for $10 \mathrm{~min}$. To the $\mathrm{KCl}, 5-10$ drops of 3:1 methanol/acetic acid fixative was added by gently mixing the plate. This solution was removed, and fresh identical fixative was added and incubated at room temperature for $5 \mathrm{~min}$. The fixation step was repeated twice and the coverslips were air-dried. After drying, coverslips were washed twice with PBS for 5 min each at room temperature. The coverslips were then incubated with Hoechst (ThermoFisher Scientific) at a final concentration of $2 \mu \mathrm{g} / \mathrm{mL}$ for $5 \mathrm{~min}$ at room temperature and washed briefly in PBS. The coverslips were then placed in $2 \times \mathrm{SSC}$ buffer and exposed to UV for 10 min followed by rinsing in $2 \times$ SSC buffer. The coverslips were then covered with $4 \%$ Giemsa stain (Gibco) for $4 \mathrm{~min}$ at room temperature. The stain was then washed off in water and the coverslips were mounted on a slide using Fluoromount G (SouthernBiotech) mounting media. Sister chromatid exchange in each group was counted per metaphase on a fluorescent microscope. At least 20 metaphases were counted per condition, and each experiment was repeated three times.

\section{Statistical analysis}

Microsoft Excel or GraphPad Prism software was used for all statistical analyses. An unpaired student $t$ test was performed on all data groups. Results were considered statistically significant when $p$ values were $<0.05$.

\section{Results}

\section{PARP1 inhibition is lethal in MPM cells}

Inhibiting PARP1 in HR-deficient cells, such as the BRCA1or 2-mutant breast and ovarian cancers, leads to an accumulation of DNA damage from replication fork collapse and ultimately cell death $[11,12]$. We tested whether the PARP1 inhibitors niraparib and olaparib could induce clonal cell death in the BAP1-mutant MPM cell lines H2452, H-Meso01A, H2461, H2731, and the BAP1 wild-type MPM cell lines CRL-2081 and H290 [7]. Olaparib and niraparib are both orally active PARP1 inhibitors that are effective in the treatment of ovarian cancers with BRCA1 and BRCA2 mutations $[18,19]$. We found that niraparib and olaparib were significantly cytotoxic to each of the MPM cell lines listed above, regardless of the status of BAP1 mutations (Fig. 1 and Supplemental Fig. S1). At the clinically relevant concentration of $3 \mu \mathrm{M}$ of niraparib, clonal survival of the MPM cell lines averaged $<10 \%$ (Fig. 1a-d). The BAP1 wildtype MPM cell line CRL-2081 was significantly less sensitive to olaparib than to niraparib (Fig. 1d, e), consistent with the benefit niraparib demonstrated in ovarian cancers that were not deficient in BRCA1 or 2 [19]. A dose response colony formation study in BAP1-mutant MPM cell line H2452 showed that the $\mathrm{IC}_{50}$ of niraparib is $400 \mathrm{nM}$ (Fig. 1f).

\section{PARP1 inhibition in MPM cells leads to replication fork arrest and collapse}

BrdU (Bromo-deoxy uridine) is a thymidine analog that is incorporated into nascent DNA during replication [14]. Active replication forks can be measured by BrdU foci using immunofluorescence. H2542 BAP1-mutant MPM cells treated with $3 \mu \mathrm{M}$ niraparib for $48 \mathrm{~h}$ and then released into fresh media containing BrdU for 30 min showed a 3.5fold decrease in BrdU foci compared to vehicle control cells (Fig. 2a). This indicated that treating MPM cells with niraparib leads to decreased replication fork restart after removal of niraparib. This implies that the replication forks were damaged beyond repair [12-14].

Stalled replication forks can eventually collapse, which is due to the loss of the replication apparatus at the fork and fork cleavage [12-14]. Collapsed replication forks after replication stress are marked by ATR phosphorylation of $\mathrm{H} 2 \mathrm{Ax}$ [14]. These increased $\gamma-\mathrm{H} 2 \mathrm{Ax}$ foci indicate that the replication fork has endured severe structural damage [20]. We found that niraparib treatment of $\mathrm{H} 2452$ cells led to a sevenfold increase in the accumulation of $\gamma$-H2Ax foci (Fig. $2 b$, supplemental Fig. S2). The increase in damaged replication forks after niraparib exposure in the MPM cells corresponds with their decreased clonal survival after niraparib.

\section{PARP1 inhibition results in genomic instability in MPM cells}

Replication fork collapse leads to chromosomal fusion events that result in mis-segregated chromosomes during mitosis $[12,14,15]$. Fused chromosomes can have two centromeres, which would result in nuclear bridging, or no centromeres, which result in the chromosome being retained as micronuclei [20]. The presence of bridging or micronuclei indicates genomic instability and mitotic catastrophe [14, 20]. Such bridging and micronuclei can be assessed using confocal immunofluorescence after DAPI-staining [14, 20]. We found that treating H2542 MPM cells with $3 \mu \mathrm{M}$ niraparib for $72 \mathrm{~h}$ generated threefold more micronuclei and twofold more bridging (Fig. 2c, supplemental Fig. S3). This was similar to the phenotype seen in other HR-deficient cancer cells $[12,14,20]$.

\section{PARP1 inhibition results in increased sister chromatid exchange in MPM cells}

Replication fork structural damage and subsequent repair often result in the exchange of DNA from one 

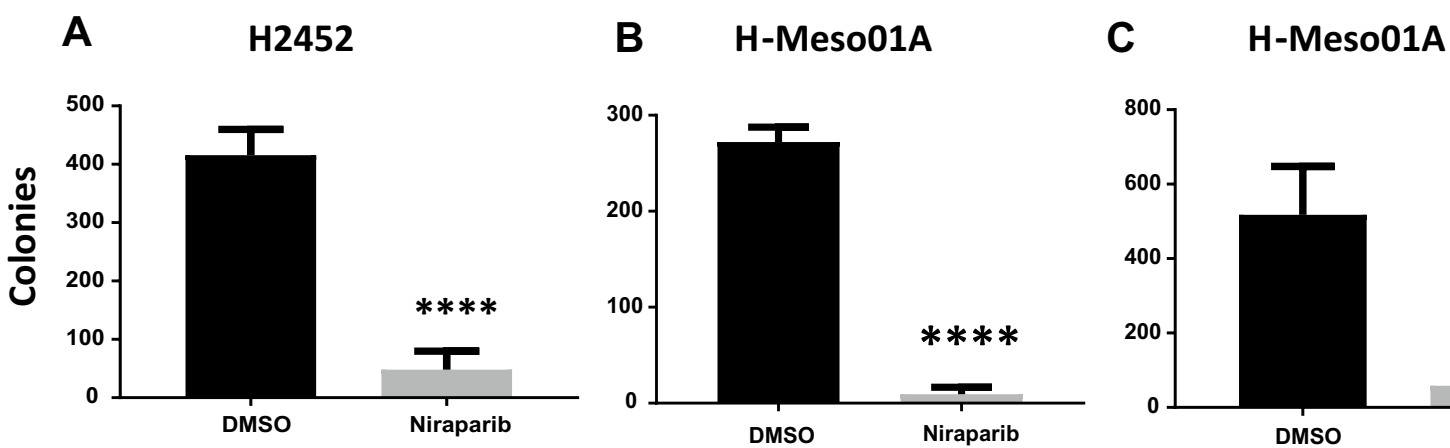

D

CRL-2081

E

CRL-2081
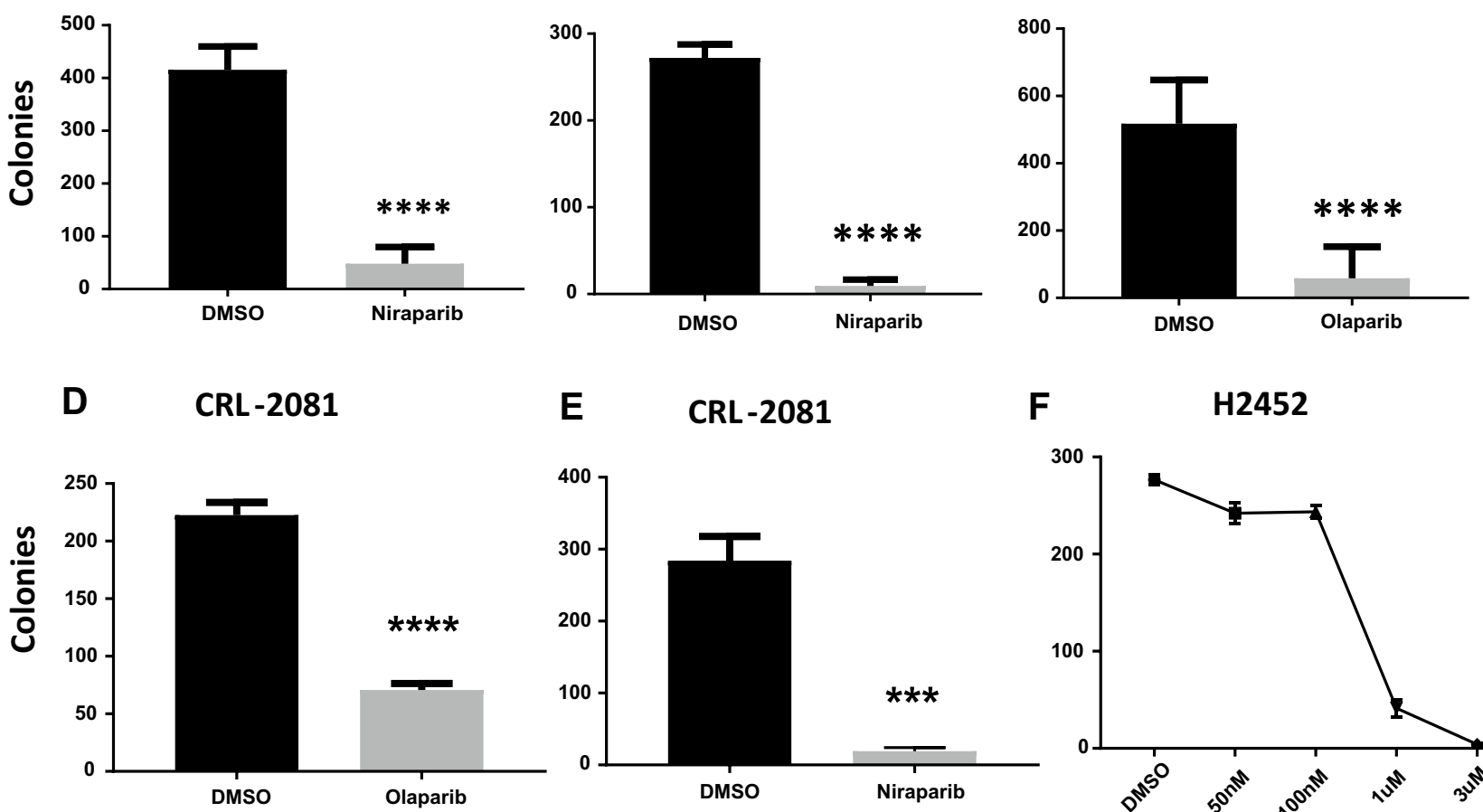

$\mathbf{F}$

H2452

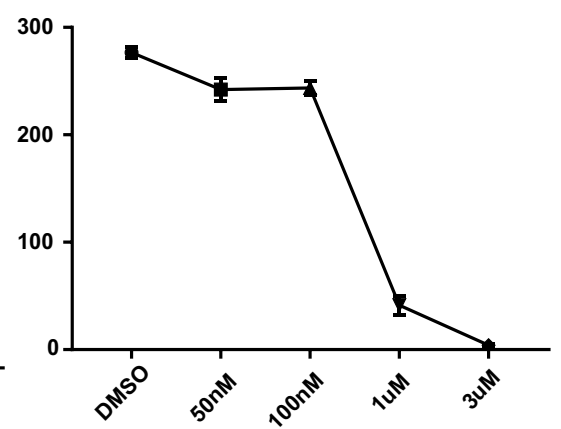

Fig. 1 PARP1 inhibition is lethal to MPM cells. Colony formation assays of clonal cell survival with continuous niraparib or olaparib, both at $3 \mathrm{uM}$. a H2452 BPA1-mutant MPM cells exposed to niraparib. b HMeso01A BAP1-mutant MPM cells exposed to niraparib. c HMeso01A BAP1-mutant MPM cells exposed to olaparib. d CRL-
2081 BAP1 wild-type MPM cells exposed to olaparib. e CRL-2081 BAP1 wild-type MPM cells exposed to niraparib. $\mathbf{f}$ Dose response of H2452 BPA1-mutant MPM cells exposed to varying concentrations of niraparib. For all figures: * indicates a $p$ value of $0.01, * *$ of 0.001 , $* * *$ of 0.0001 and $* * * *$ of $<0.0001$
Fig. 2 Replication fork and genomic instability in MPM cells after exposure to niraparib. a BRDU incorporation assays assessing replication fork repair and restart demonstrating poor fork restart after niraparib exposure in H2452 MPM cells. b Confocal immunofluorescence of $\gamma-\mathrm{H} 2 \mathrm{Ax}$ foci demonstrating a marked increase in replication fork structural damage after niraparib exposure in $\mathrm{H} 2452$ cells. c Genomic instability in BAP1-mutant mesothelioma cells after exposure to niraparib. Confocal immunofluorescence of DAPI-stained H2452 nuclei after exposure to niraparib demonstrated a significant increase in micronuclei and post-mitotic nuclear bridging. d Sister chromatid exchange (SCE) assays showing a marked increase in SCE after release from niraparib exposure in $\mathrm{H} 2452$ cells
A

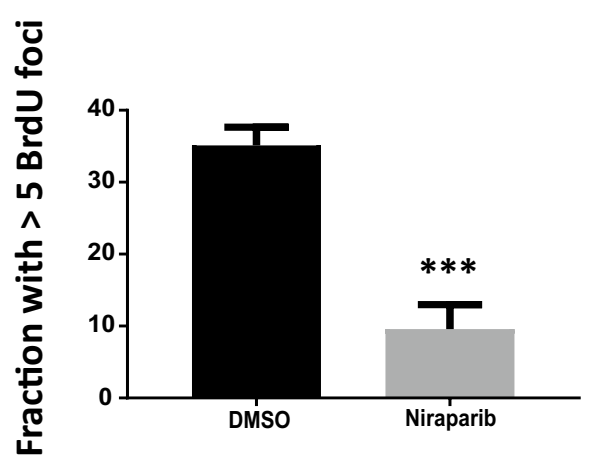

C

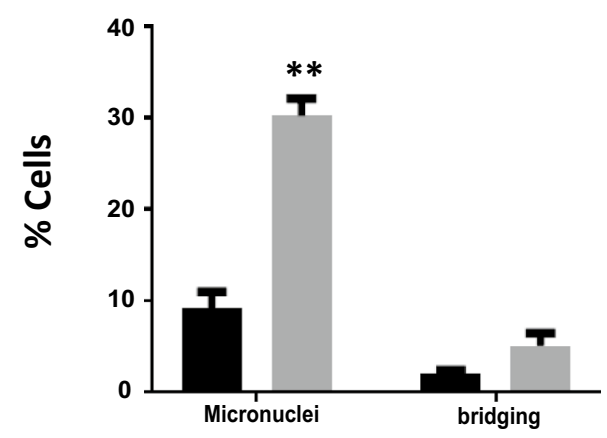

B

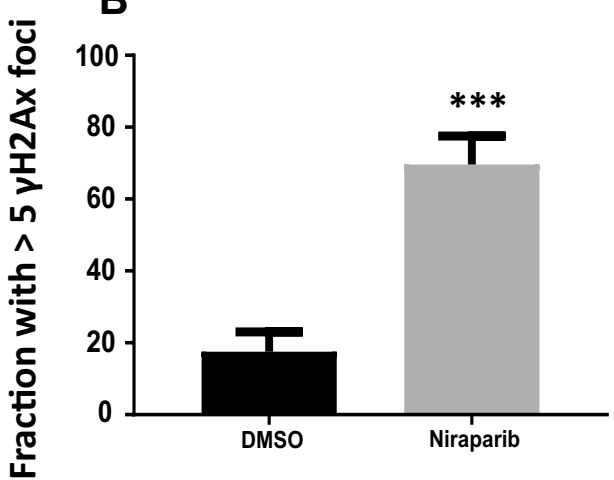

D

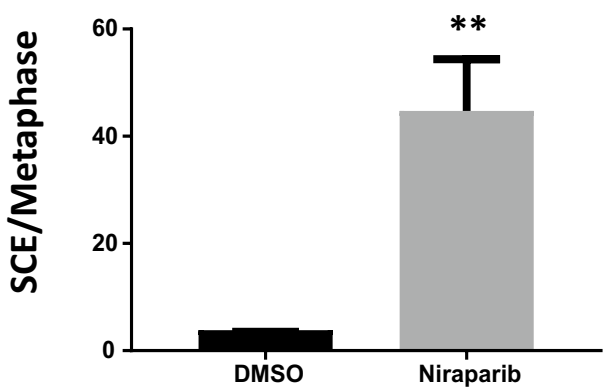


sister chromatid to another $[17,18]$. Thus, sister chromatid exchange (SCE) correlates with the rate of damaged replication forks that have attempted repair, and all replication stressing agents induce SCE. We found that the incidence of SCEs is significantly increased when H2452 MPM cells are treated with $3 \mu \mathrm{M}$ niraparib for $48 \mathrm{~h}$ and released into fresh media without the drug for $24 \mathrm{~h}$. There was a ninefold increase in SCEs in cells treated with niraparib compared to the vehicle control group (Fig. 2d, supplemental Fig. S4). This demonstrated that the niraparib-exposed MPM cells endured far more damaged replication forks than untreated cells, consistent with the $\gamma$-H2Ax data.

\section{Discussion}

MPM responds poorly to current chemotherapy or radiation therapy, and many presenting cases are unresectable due to the spread of the disease along pleural planes or chest wall invasion $[1,2]$. The average survival of patients with recurrent or unresectable disease treated with the best chemotherapy regimen (cisplatin or carboplatin plus pemetrexed) is one year [1-3]. Adding the angiogenesis inhibitor bevacizumab to this regimen adds another 6 months of survival [1, 2]. However, all unresected or recurrent patients eventually die of their disease [1-3]. Many patients with unresectable MPM choose palliative care instead of systemic chemotherapy because current regimens are difficult to tolerate [2]. Targeted inhibitors against EGFR or VEGFR have been disappointing in their clinical benefit, so there are no approved precision therapies [2]. Thus, new therapies are needed for this devastating disease.

Between 23 and $64 \%$ of malignant pleural mesotheliomas (MPMs) have somatic inactivating mutation in the BAP1 gene [4-7]. Mutation in BAP1 leads to defects in the HR DNA repair pathway, and it is considered a tumor suppressor [6-8]. The HR pathway is required for faithful repair of stalled replication forks, and HR-deficient cells become genomically unstable [12-14]. This genomic instability can result in fusion of the stalled replication forks, generating chromosomal ligations [12-14, 20]. Thus, BAP1 deficiency may be an initiating event in oncogenesis because stalled replication forks are no longer faithfully repaired due to the lack of HR [12-14]. This is similar to the inherited HR deficiencies, such as the BRCA1 and 2 mutations, that lead to breast and ovarian cancers $[12,13]$.

PARP1 inhibitors are widely used as drugs that target these BRCA1 and 2-mutant cancers [12, 13, 18, 19]. PARP1 is essential for three DNA repair pathways-single strandbreak repair, base excision repair (BER), and alternative non-homologous end joining (aNHEJ). Inhibition of PARP1 by the clinical PARP1 inhibitor drugs results in the synthetic lethal death of BRCA1 or 2 deficient cells. This cell death has multiple etiologies, all involving chromosomal instability and mitotic catastrophe [12-14, 20].

In the absence of PARP1 activity, SSBs increase in genomic DNA, either from defective SSB repair or arrested BER [13]. The unrepaired SSBs are converted into DS ends upon replication fork arrival [12]. In the absence of HR, replication fork collapse can be rescued by the aNHEJ repair pathway, but this pathway also requires PARP1 [14]. When PARP1 is inhibited in HR-deficient cells, there is an increased possibility that collapsed replication forks can be aberrantly ligated together with classical NHEJ. This leads to chromosome fusions and genomic instability, manifested by micronuclei, and bridging, as seen here [12, 20]. Another mechanism of replication fork stalling and collapse in these HR-deficient cells is that the clinical PARP1 inhibitors lock PARP1 onto DNA, blocking progression of a replication fork $[13,18]$. In that mechanism, replication forks would also be arrested at the PARP1 inhibitor-DNA complex, and that cell would lack both HR and aNHEJ activities, and thus would have great difficulty in restarting that stalled fork [12, 13, 18].

Olaparib is an oral PARP1 inhibitor that has been recently approved for the treatment of relapsed ovarian cancer that harbors BRCA1 or 2 mutations [12,13]. Niraparib is another approved potent oral PARP1 inhibitor that is well-tolerated $[18,19]$. Results of a large clinical trial using single agent Niraparib in relapsed ovarian cancer demonstrated that it was a safe and highly effective agent in ovarian cancer, regardless of HR or BRCA1/2 status [19]. The data presented here demonstrate that PARP1 inhibition using niraparib or olaparib was lethal in multiple MPM cell lines, regardless of BAP1 mutational status. The finding that niraparib decreased replication fork restart (Fig. 2a) and increased genomic instability (Fig. 2c, d) is consistent with the molecular mechanism of synthetic lethality in PARP1 inhibition in BRCA1 and 2-deficient breast and ovarian cancers $[12,13,18]$.

Bott et al. stated that they found no difference in the sensitivity to niraparib between BAP1-mutant and wildtype MPM using a cell respiration assay [7]. There was no data shown and there was no mention of whether niraparib was cytotoxic in any of the cell lines [7]. Using colony formation assays to measure cytotoxicity, which assesses not just the respiration of a cell population but clonal cell proliferative capacity, we also found that there was little difference in sensitivity to niraparib between BAP1-mutant or wild-type MPM cell lines. However, we found that niraparib was significantly cytotoxic to each MPM cell line, regardless of the BAP1 status (Fig. 1). This is consistent with a recent clinical trial where niraparib demonstrated efficacy in ovarian cancer regardless of HR status [19]. 
Olaparib also demonstrated cytotoxicity to all BAP1mutant MPM cell lines (Fig. 1, supplemental Fig. 1). In H290 BAP1 wild-type MPM cells olaparib had significant cytotoxicity, while in the CRL-2081 BAP1 wild-type MPM cells it has less benefit (Fig. 1e, f, Supplemental Fig. 1).

There are two potential reasons why both wild type and mutant BAP1 were sensitive to niraparib. First, there could be undefined HR defects in the BAP1 wild-type MPM cells. It is possible that all MPM results from HR deficiencies, with BAP1 just being the most common type of deficiency [4-7]. Second, niraparib is such a potent PARP1 inhibitor that unrepaired oxidative DNA damage leads to such massive replication fork collapse that neither an HR-deficient or replete cell can tolerate it. These two mechanisms are not mutually exclusive, and niraparib could be lethal from inhibiting BER just as much from deficient SSB repair or aNHEJ $[13,14]$.

PARP1 inhibition at clinically relevant concentrations resulting in significant cytotoxicity in MPM demonstrates that agents such as olaparib and niraparib are promising for use in the treatment of MPM, for which effective treatment is desperately needed. Thus, clinical trials of PARP inhibitors in this difficult to treat malignancy would be warranted. These data are also a further demonstration that other malignancies with acquired defects in HR may be responsive to PARP1 inhibition [8]. Studying the effects of PARP1 inhibition in the other BAP1-mutant malignancies, such as renal cell carcinoma and cholangiocarcinoma, would be especially important, since cancers also have little beneficial therapy once initial resection has failed [8].

\section{Compliance with ethical standards}

Funding This work was supported by NIH GM084020, GM109645, and CA205224 to RH.

Conflict of interest KW is employed by and holds equity in Tesaro, which owns the rights to niraparib. Tesaro did not provide any funding for this work. Every other author declares that they do not have any conflict of interest.

Human subjects This article does not contain any studies with human participants or animals performed by any of the authors.

Animal care This article does not contain any studies with animals performed by any of the authors.

Open Access This article is distributed under the terms of the Creative Commons Attribution 4.0 International License (http://creativecommons.org/licenses/by/4.0/), which permits unrestricted use, distribution, and reproduction in any medium, provided you give appropriate credit to the original author(s) and the source, provide a link to the Creative Commons license, and indicate if changes were made.

\section{References}

1. Kondola S, Manners D, Nowak AK (2016) Malignant pleural mesothelioma: an update on diagnosis and treatment options. Ther Adv Respir Dis 10:275-288. doi:10.1177/1753465816628800

2. Gomez D, Tsao AS (2014) Local and systemic therapies for malignant pleural mesothelioma. Curr Treat Options Oncol 15:683-699. doi:10.1007/s11864-014-0314-4

3. Taioli E, Wolf AS, Camacho-Rivera M et al (2015) Determinants of survival in malignant pleural mesothelioma: a surveillance, epidemiology, and end results (SEER) Study of 14,228 Patients. PLoS One. doi:10.1371/journal.pone.0145039

4. Bueno R, Stawiski EW, Goldstein LD et al (2016) Comprehensive genomic analysis of malignant pleural mesothelioma identifies recurrent mutations, gene fusions and splicing alterations. Nat Genet 48:407-416. doi:10.1038/ng.3520

5. Nasu M, Emi M, Pastorino S et al (2015) High Incidence of Somatic BAP1 alterations in sporadic malignant mesothelioma. J Thorac Oncol 10:565-576. doi:10.1097/JTO.0000000000000471

6. Testa JR, Cheung M, Pei J et al (2011) Germline BAP1 mutations predispose to malignant mesothelioma. Nat Genet 43:1022-1025. doi:10.1038/ng.912

7. Bott M, Brevet M, Taylor BS et al (2011) The nuclear deubiquitinase BAP1 is commonly inactivated by somatic mutations and 3p21.1 losses in malignant pleural mesothelioma. Nat Genet 43:668-672. doi:10.1038/ng.855

8. Kadariya Y, Cheung M, Xu J et al (2016) Bap1 is a bona fide tumor suppressor: genetic evidence from mouse models carrying heterozygous germline Bap1 mutations. Cancer Res 76:2836-2844. doi:10.1158/0008-5472.CAN-15-3371

9. Yu H, Pak H, Hammond-Martel I et al (2014) Tumor suppressor and deubiquitinase BAP1 promotes DNA double-strand break repair. Proc Natl Acad Sci USA 111:285-290. doi:10.1073/ pnas. 1309085110

10. Ismail IH, Davidson R, Gagné JP et al (2014) Germline mutations in BAP1 impair its function in DNA double-strand break repair. Cancer Res 74:4282-4294. doi:10.1158/0008-5472.CAN-13-3109

11. Fukuda T, Tsuruga T, Kuroda $\mathrm{T}$ et al (2016) Functional link between BRCA1 and BAP1 through histone H2A, heterochromatin and DNA damage response. Curr Cancer Drug Targets 16:101-109. doi:10.2 174/1568009615666151030102427

12. Shaheen M, Allen C, Nickoloff JA, Hromas R (2014) Synthetic lethality: exploiting the addiction of cancer to DNA repair. Review article. Blood 117:6074-6082. doi:10.1182/ blood-2011-01-313734

13. Wang Z, Wang F, Tang T, Guo C (2012) The role of PARP1 in the DNA damage response and its application in tumor therapy. Front Med China 6:156-164. doi:10.1007/s11684-012-0197-3

14. Wu Y, Lee SH, Williamson EA et al (2015) EEPD1 rescues stressed replication forks and maintains genome stability by promoting end resection and homologous recombination repair. PLoS Genet 11:129. doi:10.1371/journal.pgen.1005675

15. Wray J, Williamson EA, Singh SB et al (2013) PARP1 is required for chromosomal translocations. Blood 121:4359-4365. doi:10.1182/ blood-2012-10-460527

16. Gebhart E (1981) Sister chromatid exchange (SCE) and structural chromosome aberration in mutagenicity testing. Hum Genet 58:235254. doi:10.1007/bf00294917

17. Mourelatos D (2016) Sister chromatid exchange assay as a predictor of tumor chemoresponse. Mutat Res Genet Toxicol Environ Mutagen 803-804:1-12. doi:10.1016/j.mrgentox.2016.03.011

18. Jones P, Wilcoxen K, Rowley M, Toniatti C (2015) Niraparib: a poly(ADP-ribose) polymerase (PARP) inhibitor for the treatment of tumors with defective homologous recombination. J Med Chem 58:3302-3314. doi:10.1021/jm5018237 
19. Mirza MR, Monk BJ, Herrstedt J et al (2016) Niraparib maintenance therapy in platinum-sensitive recurrent ovarian cancer. N Engl J Med. doi:10.1056/NEJMoa1611310 (NEJMoa1611310)
20. Fenech M, Kirsch-Volders M, Natarajan AT et al (2011) Molecular mechanisms of micronucleus, nucleoplasmic bridge and nuclear bud formation in mammalian and human cells. Mutagenesis 26:125-132. doi:10.1093/mutage/geq052 